POLISH JOURNAL OF PHILOSOPHY

Vol. VI, No. 1 (Spring 2012), 57-68.

\title{
Cinematic Realism Reconsidered
}

\author{
Rafe McGregor \\ University of York
}

\begin{abstract}
The purpose of this paper is to re-examine the debate about cinematic motion in terms of the necessity for reception conditions in art. I shall argue that Gregory Currie's rejection of weak illusionism - the view that cinematic motion is illusory - is sound, because cinematic images really move, albeit in a responsedependent rather than garden-variety manner. In $§ 1$ I present Andrew Kania's rigorous and compelling critique of Currie's realism. I assess Trevor Ponech's response to Kania in $\S 2$, and show that his focus on the cinematic experience is indicative of the direction the debate should take. $\S 3$ demonstrates that the issue is underpinned by the question of the role of reception conditions in the experience of art. In \$4 I apply my observations on reception conditions to the problem of cinematic motion and conclude that Kania's objections are unsuccessful due to his failure to acknowledge the necessary conditions for cinematic experience.
\end{abstract}

\section{The Illusion of Realism}

Gregory Currie produced the first comprehensive philosophy of film in the analytic tradition with Image and Mind in 1995, and identified the essence of film as 'moving pictures' (Currie, 1997, p. 47). His ontology of film is strictly realist, and he rejects both cognitive and perceptual illusionism in film. In cognitive or strong illusionism the viewer believes that she occupies the position of the camera, and is therefore present in the story. Currie correctly notes that audiences neither react nor think in this way, and dismisses the 'Imagined Observer Hypothesis' (Currie, 1995, p. 167). ${ }^{1}$ In perceptual or weak illusionism, cinematic experience represents the world in a particular way, even though the viewer knows this to be false, and the thesis is that the movement of images onscreen is illusory in this sense. Currie's rejection of this claim is highly controversial, with both illusionists and realists appealing to an intuitive view of cinematic motion. In "The Illusion of Realism in Film" Kania refers to the 'common-sense' view of the illusion of movement (Kania, 2002, p. 243), and film theorists and critics seem to take it for granted that the succession of still frames in film

\footnotetext{
${ }^{1}$ Currie also refers to this hypothesis as imagining seeing.
} 
produce an illusion of continuous motion. On the other hand, Currie (1995, p. 38) maintains that the burden of proof lies with the illusionists, and his argument against perceptual illusionism is supported by aestheticians such as Berys Gaut (1998, p. 331) ${ }^{2}$ and Dominic McIver Lopes (1998, p. 347).

Although Kania endorses Currie's theory of film, and his general position on cinematic realism, he believes that the rejection of perceptual illusionism is unjustified. He begins with the accurate observation that Currie fails to present an argument for realism, merely arguing against illusionism. ${ }^{3}$ An apparently even more telling criticism is that Currie equivocates between proposing 'garden-variety motion possessed by such things as my bicycle as I ride to school' and 'special response-dependent motion' (Kania, 2002, p. 247). Garden-variety motion is characterised by an object occupying 'contiguous spatial locations at contiguous moments in time' (Kania, 2002, p. 250). Film is not analogous because there are twentyfour discrete impacts of light on the screen each second rather than a continuity of light on the screen as the images (appear to) move. In describing the cinematic image as produced by the "continuous impact of light on screen,' Currie (1995, p. 41) has thus made 'a simple factual error' (Kania, 2002, p. 250).

I am not convinced that Currie ever proposes garden-variety motion, although he does vacillate between a strong and a weak sense of "real"(Currie, 1997, p. 44). Fundamentally, he believes that the movement of images is analogous to - even like - the property of colour. Movement and colour are both real, but also dependent on a psychological reaction, and thus extrinsic (Currie, 1995, p. 32). Colours are not an illusion because I am not in error when I see red in the Tricolore. 'Roughly, for things to be red they merely have to look red to us in normal conditions' (Currie, 1997, p. 47). In discussing cinematic motion as response-dependent, Currie addresses the objection from the Müller-Lyer illusion: if cinematic motion is a 'veridical experience of a response-dependent property' a parallel argument could be constructed for the illusion created by the Müller-Lyer as veridical $(1995$, p. 44$)$. Thus the experience of the two lines 'is not the metrical property of objects we measure with rigid rods, but rather a response-dependent length,' and one's perception of this responsedependent length is regarded as veridical (Currie, 1995, p. 44). Both the Müller-Lyer illusion and cinematic motion involve perception independent of belief and the problem for Currie is that regarding response-dependent motion as real has the consequence of dismissing a paradigmatic illusion.

Currie's answer identifies the difference between the Müller-Lyer and cinematic motion as being that the appearance of the former suggests that

\footnotetext{
${ }^{2}$ Recently, however, Gaut has rejected Currie's view. See Gaut, 2010, p. 65.

${ }^{3}$ For the sake of brevity I shall henceforth use illusionism to refer to perceptual (or weak) illusionism, and realism for the opposing view.
} 
an independent check, e.g. conventional measurement, would reveal an illusion; the experience of film does not, however, suggest that an independent check-e.g. slowing down the projector speed-would reveal the movement as illusory. This is a very weak defence, because no convincing illusion draws attention to its illusory nature. Furthermore, Kania explains why slowing down the projector speed so that it produces a succession of still images is exactly analogous to measuring the lines of the Müller-Lyer:

The point is that once you have understood what is going on at the lower level of the mechanics of projection...you will change your mind about what to say at the higher level of perception of the normally projected film. (Kania, 2002, p. 249)

The Müller-Lyer is a paradigmatic perceptual illusion, and it seems as if cinematic motion belongs to the same category.

Currie's conception of the movement of images is essential to his claim that there is real movement onscreen, and is contested by Kania on the basis that Currie creates a false dichotomy. Kania agrees with Currie that there is no illusion of physical objects in motion onscreen, but rejects his alternative of a veridical experience of images in motion. 'An obvious third possibility is that we have an illusion of images moving in front of us' (Kania, 2002, p. 252). Kania believes the key to Currie's position is ascertaining whether the images themselves are reidentifiable objects, and-if so-whether they move. He refers to Currie's refutation of the argument from wave motion. This objection to realism is that the appeal to response dependence in cinematic motion has the undesirable consequence that the movement of a wave is real. There is no single, reidentifiable object in a wave, however, only a transfer of energy from molecule to molecule. Currie $(1995$, p. 45) claims that the cases are different because the body of water is a physical object which we perceive to be moving (spreading) as a wave. In cinematic motion, there is no physical object that moves, i.e. no illusion of movement; there is the real movement of an image.

Kania's concern is that Currie first disassociates the image from the object it represents in order to show that there is no illusion taking place, and then assumes that this image is 'a single reidentifiable thing' and thus not an illusion itself (Kania, 2002, p. 253). He presents a highly entertaining example of a dialogue between Paul (an illusionist) and Vince (a realist), who have just watched a film where John Wayne strides across the screen (Kania, 2002, pp. 253-254). Both Paul and Vince agree that they were watching an image of Wayne walking, but Vince holds that the image itself moved. When Paul slows the speed of the projection down, it is clear that the images are different, e.g., one shows the left side of Wayne's face and the next his whole face from the front. Vince then admits that the 
reidentifiable image itself is an illusion created by the speed of the projection, and thus that cinematic motion is an illusion of successive images. Currie's recourse to images rather than objects is therefore not enough to save his position: all movement on the screen is illusory.

\section{The Reality of Stroboscopic Motion}

Although Ponech's "External Realism about Cinematic Motion" is a response to Kania, he maintains that his view is closer to Kania's than Currie's, as he agrees that cinematic motion is external and prior to the interpretation of information by the mind (Ponech, 2006, p. 368). Ponech identifies the essence of the debate as being 'whether the image undergoes objective displacement, that is, moves continuously in a contiguous space, independent of any perceptual uptake' (Ponech, 2006, p. 354). His intended solution is extremely ambitious: to show, contra Kania and Currie, that cinematic images exhibit garden-variety motion (Ponech, 2006, p. 355).

Ponech differentiates visual displays from display formats, and demonstrates that the former possess two essential properties, pixels and stroboscopic motion. Stroboscopic motion:

denotes the continuous transformations a display undergoes, transformations partly constitutive of it. A display is more than a static field of pixels. It is also a course of events. Periods of illumination alternate with periods of nonillumination; the pixels' brightness and intensity fluctuate...This flashing is the display's stroboscopic motion. (Ponech, 2006, p. 357)

The stroboscopic motion of pixels produces and sustains cinematic imagesof-things, e.g. $W$, the image-of-John-Wayne:

$W$ is a singleton. "Singleton" designates a visible structure standing out from its background and other such structures in its vicinity. As such it is one perceptual target, perhaps among many, present in the display space. Like any singleton it is not an abstract entity but a tangible object of experience. Viewers are in sensory contact with it and have perceptual representations which are about it. (Ponech, 2006, p. 360)

The stroboscopic motion of singletons forms the basis of Ponech's theory and while he admits that singletons do not move in the same way as bicycles, he believes that they meet Kania's criteria for garden-variety motion because they occupy contiguous spatial locations at contiguous moments in time. Thus: 'The singleton, $W$, undergoes objective displacement within the visual display,' a process Ponech calls 'continuous stroboscopic displacement' (Ponech, 2006, p. 362). The claim that $W$ does not exhibit garden-variety motion is an arbitrary restriction of the term because $W$ moves as follows: 
It does not achieve objective displacement in the same way its depictum did...It does so through the mechanism of stroboscopy. $W^{\prime}$ s change of position from $L$ to $L^{n}$ is a visible, stroboscopically generated occurrent event in the display space. (Ponech, 2006, p. 365)

Singletons are real perceptual objects and their continuous stroboscopic displacement is real movement. When an observer has a cinematic experience of a singleton in motion, therefore, there is no illusion involved.

"Singletons" are Ponech's technical term for Currie's identification of an image of a particular as a 'particular reidentifiable thing' (Currie, 1996, p. 340). Currie claims that there are 'images of reidentifiable physical objects moving in front of our eyes,' as opposed to the objects themselves (Currie, 1996, p. 338). (The exception is, of course, animation, where the images are not of physical objects. ${ }^{4}$ ) Cinematic technology produces the particular reidentifiable thing onscreen, and Currie believes that this thing really moves:

We see a cinematic image of a man, and we see that it is in one place on the screen, and we later see that it is in another; indeed, we see that image move from one place to another on the screen. (Currie, 1995, pp. 40-41)

I shall henceforth use Ponech's term when discussing this type of image. Kania denies the existence of singletons, ${ }^{5}$ and I present the three characterisations of cinematic motion as follows:

Ponech: the motion of singletons is objectively real.

Currie: the motion of singletons is subjectively real (because it is response-dependent, like colour).

Kania: singletons and cinematic motion are illusory (and thus responsedependent).

I do not wish to discuss the ontological status of singletons in detail, so I shall restrict my observations to the obvious: if one considers their reality in terms of existence and independence, they are different to bicycles. In his description of stroboscopic motion, Ponech notes: 'This type of motion is not to be confused with objective displacement. Pixels do not roll across the screen like billiard balls' (Ponech, 2006, p. 357). But the motion of bicycles and billiard balls is exactly the kind of motion to which Kania is referring when he discusses garden-variety motion. He is quite clear that for 'normal' or garden-variety motion there must be: (1) an object, and (2) that object must occupy contiguous spatial locations at contiguous moments in time (Kania, 2002, p. 250). The absence of the object is Kania's reason for

\footnotetext{
${ }^{4}$ See: Currie 1995, p. 41; Currie 1996, p. 340; and Kania, 2002, p. 252.

${ }^{5} \mathrm{I}$ address his objection in $\S 4$.
} 
claiming that there is no garden-variety movement in the cinema, as opposed to the theatre (Kania, 2002, p. 248).

Singletons do move by occupying contiguous spatial locations at continuous moments in time. They are not, however, objects. And because they are not objects they do not move objectively, as Ponech states. I present three examples, applied to the motion of objects and singletons respectively:

(1) I watch Dr Kania cycle to school.

(2) My dog watches Dr Kania cycle to school.

(3) Dr Kania cycles to school.

(1*) I watch my television screen while $W$ moves across it.

(2*) My dog watches my television screen while $W$ moves across it.

(3*) $W$ moves across my television screen.

What happens in (1) to (3)? There is garden-variety motion in all cases: an object, a man on his bicycle, moves contiguously. At a particular point in time, man and bicycle are at home; at a subsequent point in time, man and bicycle are at school. The presence or absence of observers human or canine makes no difference to the sequence of events that result in the object arriving at school. In ( $1 *)$, I watch $W$ move contiguously. It is not clear what happens in $\left(2^{*}\right)$, suffice to say that my dog does not perceive $W$. All we can really say about $\left(2^{*}\right)$ and $\left(3^{*}\right)$ therefore, are that pixels (really) flash. Like Locke's secondary qualities these flashing pixels have the power to produce $W$ in a being which has the relevant perceptual apparatus. But the sequence of events that take $W$ from one side of the screen to the other only occurs when it is perceived by me. When I am present, pixels flash and $W$ moves across the screen; when I am absent, pixels flash.

While Ponech has shown that singletons move in the same way as objects - contiguously in space and time- - he cannot show that they exhibit garden-variety motion because they are not objects. His reference to a singleton as a 'perceptual object' reinforces the point (Ponech, 2006, p. 367). A singleton is a perceptual object only for beings that possess the appropriate perceptual apparatus. I have that apparatus, my dog does not. My dog can see the bicycle, but does not see the same colour as I see. Ponech notes that singletons are not abstract entities but tangible objects of experience. The unstated difference here is between tangible objects and tangible objects of experience. Tangible objects like bicycles appear to have an independent existence, but tangible objects of experience like singletons appear to remain a potentiality until a creature with the matching perceptual apparatus experiences them. In Ponech's terms, therefore: $W$ 's movement is internal; the flashing pixels are external.

It seems as if the debate, which is a very specific issue within a subcategory of philosophy of art, has exhausted itself. I think there is more to 
say, however, and that Ponech indicates the direction the enquiry should take when he denies that Kania's slowing down of the film projector demonstrates illusionism:

Reductionism thus mistakenly believes we glimpse cinema's true nature by slowing or freezing the exhibition machinery. All we thereby glimpse is a quasi-cinematic display momentarily deprived of one of its ontological primitives. (Ponech, 2006, p. 359)

The second sentence suggests that a necessary condition for cinematic experience is absent. In what follows, I shall focus on film and music, although - as my brief examples of literature and painting show - the issue of reception conditions is relevant to all art.

\section{Reception Conditions for Art Forms}

Kania's argument against Currie relies upon a single contention, the connection between the succession of still images that constitute the medium of film, and the perception of the moving images on the screen. $\mathrm{He}$ summarises his position: 'Yet again we can see that film's frame-by-frame nature forces us to deny that its images really move, in any standard sense' (Kania, 2002, pp. 250-251). This is simply not the case, however, as I shall demonstrate. Kania makes an error when he states that 'the number of frames per second is irrelevant to the necessary condition of paradigmatic movement' (Kania, 2002, p. 257). There is no mystery or trickery in film projection: static frames are projected at the speed of twenty-four frames per second (fps) because this is the optimum speed at which human beings perceive motion onscreen. This perception is caused by persistence of vision and the phi phenomena. The former consists in the brain's retention of images on the retina for a fraction of a second after the removal of the stimulus; the latter is the phenomenon that causes the rotating blades of a fan to appear as a complete circle at certain speeds. The speed of projection thus operates so as to guarantee that the audience will experience a film and not a slide show, and won't see dark intervals between the frames. Projection at $24 \mathrm{fps}$ is therefore a necessary condition for the reception of the art form of film by an audience.

I propose an analogy with a non-visual art, namely music. I can experience, e.g., Holst's The Planets in a number of ways, from attending a live performance to playing a record or $\mathrm{CD}$, or downloading it onto my MP3 player. In each case, there are various factors that count towards me experiencing Holst, such as the instruments used to produce the music and the ability of the musicians to follow the score faithfully. If I attended a performance where "Mars, the Bringer of War" was played on a didgeridoo, I could not be said to have experienced Holst. Nor would I have the 
experience if I played a faulty CD where all I could hear was the whirring of the disk in the player. I would not have the musical or aesthetic experience of Holst if I played a 331/3 revolutions per minute (rpm) record of The Planets at $45 \mathrm{rpm}$ either. I would hear sounds, perhaps even music, but not Holst, and certainly not have an aesthetic experience.

The presentation and appreciation of art involves different types of media and different reception conditions. Art forms are not necessarily restricted to a particular medium, but whatever medium is used determines the necessary conditions for an audience to be able to experience the work. It seems that many-if not most-of these are so obvious that they frequently escape notice. Remaining with non-visual art, I would not have the experience of James Joyce's Ulysses if I read a word a day. In fact, even at the rate of a word an hour, every hour, the novel would take about thirty years to complete. The literary experience differs for each person, but there is a requirement that a work be consumed regularly, and with short enough intervals that one's memory and understanding can function appropriately. Visual arts have similarly obvious reception conditions: I wouldn't be able to fully appreciate Picasso's Les Demoiselles D'Avignon if I lay on the floor to look at it, or if the light was too dark for me to make out the depiction. Reception conditions for the experience of paintings require sufficient light and a view with as little of an angle as possible, i.e. from directly in front, at approximately eye-level.

I think the analogy between film and music is forceful. First, I can have the aesthetic experience of Orson Welles' Citizen Kane in a number of ways, and this experience is not reliant upon a specific medium. I can attend a performance of the film, watch it on video on my television, or watch it on my computer on the train. As with a musical performance, certain reception conditions must prevail in order for me to have the experience, and there may be arguments advanced as to the ideal conditions for the ideal experience, e.g. watching the film in a theatre, where the light is at an optimum level and the screen is an optimum size. Second, there are circumstances where I will not actually have the Citizen Kane experience. Significantly, those circumstances include watching the film at $1 \mathrm{fps}$ and 48 fps: in fact, at any speed other than the $24 \mathrm{fps}$ at which it was designed to be shown. I did not experience Holst at $45 \mathrm{rpm}$, and I do not experience Welles at 45 fps. Ponech's description of a quasi-cinematic display is therefore entirely accurate.

The experience of film is the experience of seeing moving images under specific perceptual circumstances (e.g., 24 fps on a white screen in a dark room), which are necessary for the experience. This conception of the cinematic experience results in a serious problem for Kania. As the art form is independent of the specific medium used in production, the nature of cinematic motion must be considered independently from the means used to 
produce the motion. The view that the illusory nature of film can be proved by slowing the projector down is thus doubly fallacious: because there are - or could be - other methods of producing the work, and because once the conditions for aesthetic experience and perception are changed, one can no longer be said to experience the work of art. With this in mind, I return to Currie's theory of perceptual realism.

\section{The Myth of Illusion}

I agree with Kania's scepticism of claims that put the burden of proof on the opposing argument, but Currie seems correct in this instance. If one is considering perception in general, or any particular aspect thereof, the default setting is that humans with fully functioning senses perceive reality rather than illusion. If you want me to believe that the lines in the MüllerLyer illusion are equal in length, then you must demonstrate the fact. It looks like the one is longer than the other, and until the illusion is revealed to me, I assume my perception is veridical. Similarly, it seems to me that film consists of moving pictures. If the pictures do not move, then I require convincing. While I acknowledge that Currie's failure to provide an argument for the reality of movement is indeed a weakness, I still accept that it is the illusion that requires proof.

In Image and Mind Currie's 'view is simply that cinematic motion is real in the weak sense,' meaning that the movement is real as opposed to illusory (Currie, 1995, p. 44). He thus accepts that cinematic motion is not real in the strong sense, where real is contrasted with illusory and apparent. Subsequently, however, he admits to being unable to decide which sense of real is applicable to cinematic motion, and offers support for both (Currie, 1997, p. 47). All of Currie's arguments rely on the comparison of the reality of cinematic movement with the reality of colour, but the suggestion that the movement is real in the strong sense is an alignment with Ponech's external realism. In perceiving motion onscreen one would not normally admit to being deceived (as by the Müller-Lyer illusion), but one might well think that the movement, like the colours, was apparent rather than independently real. I have rejected Ponech's theory in $\S 2$, and I shall therefore interpret Currie's thesis as allowing for real movement in the weak sense, i.e. that movement is real, but an instance of responsedependent movement.

In this case, Kania's objection to Currie's handling of the Müller-Lyer counter-example must be addressed. An uninformed glance at the MüllerLyer wouldn't provoke a desire to measure the lines any more than watching a film provokes the desire to slow down the projection speed. If, however, I decide that I require an independent check, then-as Kania states - slowing down the projection speed seems entirely appropriate. Such 
a check would indeed show that the work of art I am appreciating is produced by the projection of a series of static images, but the act of checking (the slowing down of these images) would mean a departure from the appreciation of the work itself. Measuring the Müller-Lyer lines shows that the illusion is produced by the arrows, and slowing down the rate of projection shows that a film is produced by a series of static images. But Currie, Kania, and Ponech are concerned with the art of film and that art involves certain necessary reception conditions, one of which is projection at $24 \mathrm{fps}$. Watching the film at a different speed may be educational or entertaining, but is no longer an experience of the work of art.

In addressing the counter-example of the wave, Currie holds that where the body of water is a physical object that appears to move, there is no physical object in cinematic motion, only a singleton. Kania rejects this concept. In his dialogue, he has Paul present John Wayne frame-by-frame to show that the image is different in each frame. I cannot accept this argument because it involves a departure from the reception conditions of the film, but I offer another on Kania's behalf. There is a particularly effective sequence of about thirty seconds in Agustín Díaz Yanes' Alatriste (2006), where Captain Alatriste meets his archenemy, Gualterio Malatesta. The scene begins with an oblique view of a narrow alley: Alatriste enters bottom right and walks away from the camera. He stops and turns back to the camera, whereupon the view changes to a shot of his body from the front, with the camera zooming slowly in to his face. The audience then sees Alatriste's point of view as a herd of sheep trot around the corner. The shot returns to Alatriste's face, follows his hand as he draws his dagger, and the prologue to Malatesta's entrance finishes with a close-up of the tension on Alatriste's face. The image at the beginning, of Alatriste's broadbrimmed hat and cloak seen from behind and above, is completely different from the close-up frontal shot of his face at the end. Although the sequence either follows Alatriste or his point of view throughout, it clearly begins and ends with two different images. Kania claims that images are not singletons, and cannot therefore really move.

The experience of film, however, provides evidence to the contrary. When I watch the above sequence, I don't see Viggo Mortensen onscreen because the camera is not transparent. I see a two-dimensional cinematic representation of Mortensen (and Captain Alatriste), and this representation takes the form of an image that changes hundreds - or perhaps thousandsof times during the film. As Currie notes, "no particular light wave or particle is more than minutely constitutive of it' (Currie, 1995, p. 41). I wasn't confused when the camera switched from the view of Mortensen's silhouette emerging from the shadows to a close-up of his face because I recognised the image of the actor throughout; this recognition occurs because the representative image is reidentifiable. In motion pictures-as 
opposed to photographs - there is no paradox in the image both changing and remaining the same. If I am shown two photographs of John Wayne taken from different angles, I will call them different. Show me a film where he strides across the screen, and I will say that the singleton $W$ moved. Slow the film down or speed it up as much as you like, but if we're talking about cinema then I expect images to move, and movement means change.

The point is that the discussion is about film and Currie has already identified film as essentially moving pictures. Definitions may differ, but there is general agreement that film consists of a depiction of some sort (be it a shadow, photograph, picture, or image) which moves, ${ }^{6}$ i.e. depiction and motion are essential to the art form. Kania's objection fails because cinematic motion is the real movement of singletons. This movement is apparent (i.e., response dependent), but it is not illusory (i.e., deceptive). The fact that cinematic motion is produced by static frames shows only that a certain speed of projection-24 fps - is a reception condition of the art form. Once one departs from that projection speed, one is no longer experiencing the art form, which is where the analogy with the Müller-Lyer illusion breaks down. Ponech's attempt to prove that singletons exhibit garden-variety motion also fails. They may indeed move contiguously in space and time, but they are not objects and differ from objects in that their existence is dependent on perception. I conclude, therefore, that Currie's theory of cinematic motion is the most plausible of the three options.

\section{References}

Carroll, N. (1996). Defining the Moving Image. In Carroll, N., \& Choi, J. (Eds.), Philosophy of Film and Motion Pictures (113-133). Oxford: Blackwell.

Currie, G. (1995). Image and Mind: Film, Philosophy, and Cognitive Science. Cambridge: Cambridge University Press (2008 edition).

Currie, G. (1996). Film, Reality, and Illusion. In Bordwell, D., \& Carroll, N. (Eds.), Post-Theory: Reconstructing Film Studies (325-344). Madison: The University of Wisconsin Press.

Currie, G. (1997). The Film Theory that Never Was: A Nervous Manifesto. In Allen, R., \& Smith, M. (Eds.), Film Theory and Philosophy (42-59). London: Oxford University Press, 1997.

Gaut, B. (1998). Imagination, Interpretation, and Film. Philosophical Studies, 89 (2/3) 331-341.

\footnotetext{
${ }^{6}$ Noël Carroll notes several marginal cases in film where images do not move, but even if one accepts these there must be the potential for movement. See: Carroll, 1996, p. 125.
} 
Gaut, B. (2010). A Philosophy of Cinematic Art. Cambridge: Cambridge University Press.

Kania, A. (2002). The Illusion of Realism in Film. British Journal of Aesthetics, 42 (3), 243-258.

Lopes, D.M. (1998). Imagination, Illusion and Experience in Film. Philosophical Studies, 89 (2/3), 343-353.

Ponech, T. (2006). External Realism about Cinematic Motion. British Journal of Aesthetics, 46 (4), 349-368. 